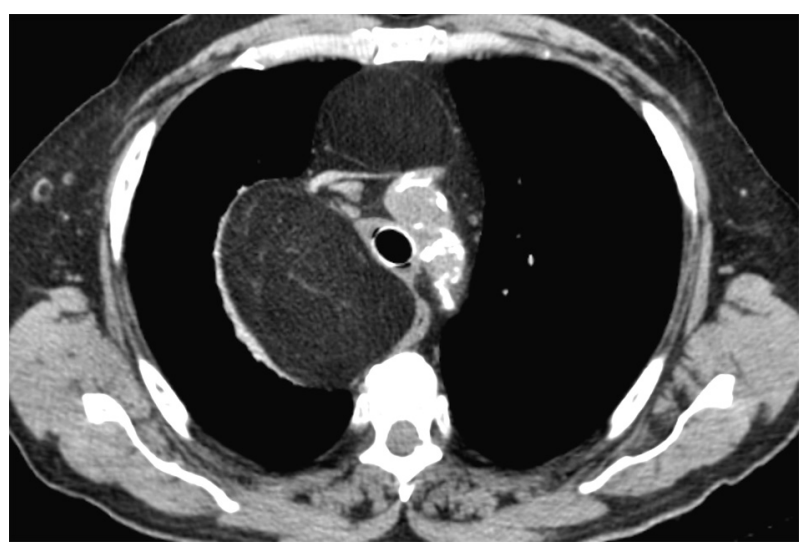

FIGURE 2. Computed tomographic image showing the endotracheal stent in place allowing complete patency of the airway after 4 years.

to compression may occur. Symptoms related to lung or airway compression include cough, chest pain, dysphonia, and even significant dyspnea and stridor. Episodes of asphyxia or respiratory insufficiency consequent to critical tracheal or bronchial stenosis, however, have been reported only as anecdotal findings. ${ }^{4,5}$

In cases of asymptomatic disease, no operative treatment is indicated; when symptoms related to mass effect are present, however, complete surgical resection or debulking can be required. ${ }^{1,3}$ In some cases related to exogenous steroid excess, symptomatic relief can be obtained by the simple reduction of the steroid consumption. ${ }^{5}$
In the presence of significant airway compression, stenting of the restricted lumen may be a viable alternative justified by the benign behavior of the lipomatous mass, with slow growth and soft consistency. Very limited data have been reported in the literature, however, to assess the reliability of this therapeutic option at long term.

Durable efficacy of the endoscopic treatment reported in this case proves that when the critical mass effect is limited to the trachea and surgical resection poses a high risk, restoration of respiratory wellness can be obtained by simple airway stenting. This case report challenges the widely held concern that long-term stents should not be used in the setting of benign disease.

We thank Dr Marta Silvi for data management and editorial work.

\section{References}

1. Nguyên KQ, Hoeffel C, Lê LH, Phan HT. Mediastinal lipomatosis. South Med J. 1998;91:1169-72.

2. Mohapatra PR, Janmeja AK. Images in clinical medicine. Asymptomatic mediastinal lipomatosis. N Engl J Med. 2010;363:1265.

3. Peek DF, Heijmen RH, Ernst SM, Schepens MA. Extensive mediastinal lipomatosis in a patient with severe aortic valve stenosis. Eur J Cardiothorac Surg. 2002;21: 564-5.

4. Hoskins MC, Evans RA, King SJ, Gishen P. 'Sabre sheath' trachea with mediastinal lipomatosis mimicking a mediastinal tumor. Clin Radiol. 1991;44:417-8.

5. Sorhage F, Stover DE, Mortazavi A. Unusual etiology of cough in a woman with asthma. Chest. 1996;110:852-4.

\title{
An unusual presentation of esophageal metastasis from breast cancer
}

\author{
Megan A. Wilson, MD, ${ }^{\mathrm{a}}$ Nilay Shah, MD, MS, ${ }^{\mathrm{b}}$ Mark E. O’Donnell, MD, FRCS, ${ }^{\mathrm{b}}$ and \\ Dawn E. Jaroszewski, MD, FACS, ${ }^{a}$ Phoenix, Ariz
}

Breast cancer metastases to the esophagus are rare, affecting $0.6 \%$ of patients with breast cancer. ${ }^{1}$ This rarity may present a diagnostic challenge related to the

From the Division of Cardiovascular and Thoracic Surgery, ${ }^{\mathrm{a}}$ Mayo Clinic Hospital, Phoenix, Ariz; and the Department of Surgery, ${ }^{\mathrm{b}}$ Mayo Clinic Hospital, Phoenix, Ariz.

Disclosures: Authors have nothing to disclose with regard to commercial support.

Received for publication May 10, 2014; revisions received Feb 5, 2015; accepted for publication Feb 19, 2015; available ahead of print March 24, 2015.

Address for reprints: Dawn E. Jaroszewski, MD, FACS, Division of Cardiovascular and Thoracic Surgery, Mayo Clinic, 5777 E Mayo Blvd, Phoenix, AZ 85054 (E-mail: drshahnilay@ gmail.com).

J Thorac Cardiovasc Surg 2015;149:e110-2

$0022-5223 / \$ 36.00$

Copyright (c) 2015 by The American Association for Thoracic Surgery

http://dx.doi.org/10.1016/j.jtcvs.2015.02.038 infrequency and the lengthy disease-free survival often seen before metastatic presentation. We present a rare case report of esophageal metastasis from breast cancer after a 13-year disease-free interval.

\section{CLINICAL SUMMARY}

A 62-year-old woman was seen at our institution for consideration of esophagectomy as the result of a recalcitrant esophageal stricture noted to be benign on biopsy. She had experienced significant dysphagia to all but liquids since 2007 and during that interval had a 60-pound weight loss despite monthly esophageal dilations. The patient had a previous history of left-sided lobular breast cancer treated with lumpectomy, axillary dissection, and adjuvant chemoradiation therapy in 1994 . The stricture was 


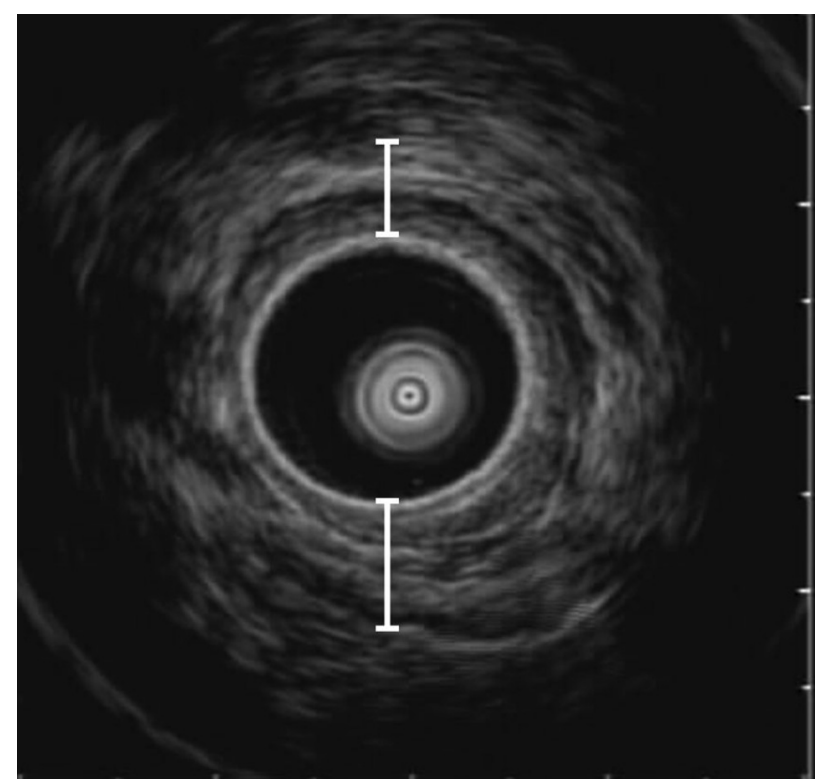

FIGURE 1. Endoscopic ultrasonography demonstrating a tight fibrotic stricture at $26 \mathrm{~cm}$ where all layers of the esophagus were indistinct from each other, with the muscularis propria appearing extremely fibrotic.

presumed to be secondary to radiotherapy administered for treatment of her left-sided breast cancer in 1994. Previous esophageal biopsies on multiple occasions had demonstrated benign squamous cells with additional atypical glandular cells. An $18 \mathrm{~mm} \times 15 \mathrm{~cm}$ Polyflex esophageal stent (Boston Scientific Corporation, Marlborough, Mass) had been previously deployed for a 7-week period, with a corresponding improvement in swallowing. The stent was removed after 7 weeks because her swallowing had improved but never seemed to be normal.

Endoscopic ultrasonography demonstrated a 5 -cm tight fibrotic stricture starting at $26 \mathrm{~cm}$ where all layers of the esophagus were indistinct from each other. The muscularis propria appeared extremely fibrotic (Figure 1). Additional biopsy specimens were taken and again showed no malignancy. Because of her recalcitrant but apparently benign symptomatic stricture, she was offered and then scheduled for a minimally invasive esophagectomy, a procedure that is based on a minimally invasive Ivor Lewis procedure and has been previously described by our own unit. ${ }^{2}$

Histopathologic analysis confirmed an invasive adenocarcinoma in the muscularis propria of squamous-lined esophagus, with tumor morphology compatible with a breast primary cancer that was also estrogen receptor positive. Human epidermal growth factor receptor was negative (Figure 2).

The patient had an uneventful perioperative recovery. The patient's feeding tube was removed after confirmation of completed healing. Oncology initiated anastrozole for adjuvant therapy. At 5 years of follow-up, the patient has remained well without evidence of recurrence.

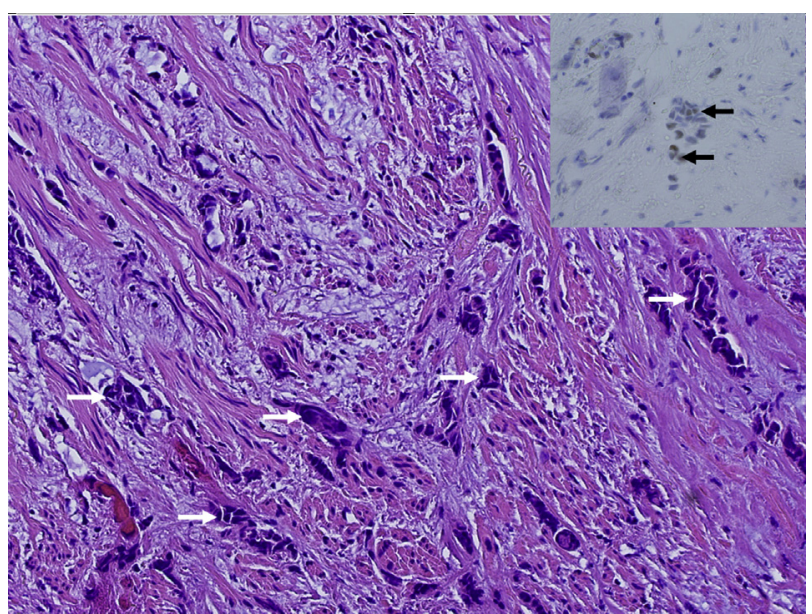

FIGURE 2. An invasive adenocarcinoma in the muscularis propria of squamous-lined esophagus with tumor morphology compatible with a breast primary cancer that was also estrogen receptor positive. Arrows designate clusters of adenocarcinoma.

\section{DISCUSSION}

Breast cancer is the most common female cancer, affecting 1 in 8 American women with 39,620 deaths reported annually. Death rates from breast cancer have been declining during the last 25 years, presumably related to earlier detection through screening programs combined with improved surgical and oncologic treatment modalities. The most common sites of metastatic spread are bone, liver, brain, and lung, whereas gastrointestinal metastasis remains rare. Most tumors metastasizing to the gastrointestinal tract are lobular breast carcinomas, which have a predilection toward the stomach and colon. ${ }^{1,3}$ Secondary involvement of the esophagus is only reported in $1 \%$ to $6 \%$ of cancer cases. Most common cancers metastasizing to the esophagus are lung and breast cancers. Other primary sites can be kidney, pancreas, ovary, thyroid, bladder, rectum, thyroid, liver, and endometrium. We report an unusual case of metastatic breast cancer to the esophagus diagnosed after esophagectomy.

Reported presentations of metastatic breast cancer after treatment of the primary breast cancer have ranged from 8 to 30 years. Our patient had symptoms after 13 years. A similar case report has been published in which clinical symptoms and radiographic imaging mimicked radiation-induced stricture. The authors of that report emphasize the rarity of such a lesion, which may indeed become more common with improved long-term survival after initial breast cancer treatment. ${ }^{5}$ Our patient was recommended for esophagectomy solely because of the diagnosis of benign stricture leading to prolonged dysphagia. Breast cancer metastasis was an incidental finding that was not even presumed in our differential diagnosis.

Our case highlights some of the diagnostic challenges attributed to chronic esophageal strictures, with which patients often have a history of multiple normal esophageal 
biopsy results. Rampado and colleagues ${ }^{1}$ have previously reported the "breast-esophagus" syndrome in patients with breast cancer with deteriorating dysphagia, macroscopically normal esophageal mucosa, and benign endoscopic biopsy results for whom minimally invasive resection modalities are suggested if technically feasible. Therapeutic interventional strategies tend to target symptomatic palliation, rather than curative resection. Curative resection in our case was incidental.

In conclusion, a possibility of gastrointestinal metastasis should be kept in the differential diagnosis in any patient with history of breast cancer who is seen with esophageal stricture and new gastrointestinal symptoms.

\section{References}

1. Rampado S, Ruol A, Guido M, Zaninotto G, Battaglia G, Costantini M, et al. Mediastinal carcinosis involving the esophagus in breast cancer: the "breast-esophagus" syndrome: report on 25 cases and guidelines for diagnosis and treatment. Ann Surg. 2007;246:316-22.

2. Laxa BU, Harold KL, Jaroszewski DE. Minimally invasive esophagectomy: esophagogastric anastomosis using the transoral Orvil for the end-to-side Ivor-Lewis technique. Innovations (Phila). 2009;4:319-25.

3. Varanasi RV, Saltzman JR, Krims P, Crimaldi A, Colby J. Breast carcinoma metastatic to the esophagus: clinicopathological and management features of four cases, and literature review. Am J Gastroenterol. 1995;90:1495-9.

4. Haney JC, D'Amico TA. Transhiatal esophagogastrectomy for an isolated ovarian cancer metastasis to the esophagus. J Thorac Cardiovasc Surg. 2004; 127:1835-6.

5. Nazareno J, Taves D, Preiksaitis HG. Metastatic breast cancer to the gastrointestinal tract: a case series and review of the literature. World J Gastroenterol. 2006; 12:6219-24. 